\title{
Nonrigid Registration of Dynamic Renal MR Images Using a Saliency Based MRF Model
}

\author{
Dwarikanath Mahapatra and Ying Sun \\ Department of Electrical and Computer Engineering, National University of \\ Singapore, 4 Engineering Drive 3, Singapore 117576, Singapore \\ \{dmahapatra, elesuny\}@nus.edu.sg
}

\begin{abstract}
Nonrigid registration of contrast-enhanced MR images is a difficult problem due to the change in pixel intensity caused by the washin and wash-out of the contrast agent. In this paper we propose a novel saliency based Markov Random Field approach for effective nonrigid registration of contrast enhanced images. Saliency information obtained from the neurobiology-based saliency model alongwith intensity information is used to quantify the degree of similarity between images in the pre- and post-contrast stages. Information from these two features is combined by using an exponential function of the saliency difference such that it assigns low values to small differences in saliency and at the same time ensures that saliency information does not bias the energy term. Rotationally-invariant edge information from edge-orientation histograms was used to complement the saliency information resulting in better registration results. Tests on real patient datasets show that our algorithm results in accurate registration. We also simulated elastic motion on images, and the deformation field recovered by our algorithm was nearly the inverse of the simulated field.
\end{abstract}

\section{Introduction}

Nonrigid image registration, also referred to as warping, is an essential step in medical image analysis. Over the years, a number of methods have been proposed to meet the challenges arising from registration of images where the object of interest has been elastically deformed. Some of the earliest methods include elastic models [1, fluid flow models [2]3] and an optical flow approach [4. In [5] the registration problem has been solved using thin-plate splines where the images are deformed over a regular grid having numerous control points. The disadvantage of thin-plate splines is that they have a global region of support i.e., changing the position of one control point changes the entire deformation field. Rueckert et al. in [6] overcome this problem by using B-splines which have a local region of support. Rohde et al. in 7 introduce the adaptive bases algorithm which uses radial basis functions instead of traditional B-splines and allows for spatial adaptation of the displacement field in regions of misregistration only.

Recently, Markov random field (MRF) model has also been used to achieve nonrigid image registration. In an MRF formulation of the problem of nonrigid

D. Metaxas et al. (Eds.): MICCAI 2008, Part I, LNCS 5241, pp. 771779, 2008.

(C) Springer-Verlag Berlin Heidelberg 2008 
image registration, the solution is obtained as the maximum a-posteriori (MAP) configuration or equivalently, by minimizing the energy of the corresponding MRF. Roy and Govindu in 8], applied an MRF model for the optical flow problem by modeling the flow orientation and magnitude as separate fields. The $\alpha-\beta$ swap algorithm proposed by Boykov et al in [9] used graph cuts to find the minimum of an energy function in the case of 2D motion estimation. Shekhovtsov et al. in 10 have proposed an algorithm that uses blocks of pixels to effectively register non-rigid deformations in synthetic and real images and is computationally more tractable. Tang et al. in [11] use an MRF based model to elastically register brain images. In [12, to avoid the effects of contrast enhancement on the process of image registration, Zheng et al. propose an MRF framework to de-enhance dynamic contrast-enhanced MRI and then register them using the B-Spline based algorithm in [6].

In this paper, we propose a novel saliency based approach for the nonrigid registration of dynamic renal MR images that can effectively correct elastic deformations in the presence of contrast enhancement due to wash-in of the contrast agent. In our approach, nonrigid registration is achieved by integrating saliency and edge information alongwith pixel intensity into the MAP-MRF framework. Saliency information of the pre- and post-contrast stages combined with pixel intensity are used to quantify the degree of similarity between two regions in the presence of intensity changes. By the use of orientation histograms, edge information has been used to complement saliency information.

The rest of the paper is organized as follows. In Section 2 we describe the neurobiology-based saliency model and its advantage over an entropy-based saliency model. Section 3 describes our saliency based MRF model and its optimization. We present our results in Section 4 and conclude with Section 5

\section{Neurobiology-Based Saliency Model}

Saliency is a concept which states that there are regions in a scene that are more "attractive" than their neighbors and hence draw attention. Fig. 1(a)-(c) show respectively the cropped image corresponding to the right kidney (of the patient) in one slice, the saliency map generated by an entropy-based approach 13], and by a neurobiology-based approach [14. The saliency map shown in Fig. 1 (b) gives a lot of importance (in terms of saliency values) to less important regions surrounding the kidney. In contrast, the saliency map in Fig. 1 (c) shows distinct salient regions corresponding to the kidney. Therefore, we opt for the neurobiology-based saliency model described in [14] and use luminance and edge information to calculate the saliency.

Saliency Map for Pre- and Post-Contrast Enhanced Images: In dynamic contrast enhanced images, the intensity of certain areas in the image changes with time (see Fig. 1 (d) for a post-contrast image of the same kidney shown in Fig. 1 (a)). By increasing the intensity of an already salient region, the saliency map does not change. Saliency being a measure of how one region differs from its 


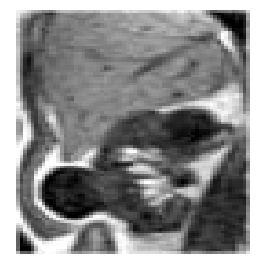

(a)

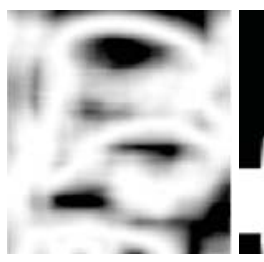

(b)

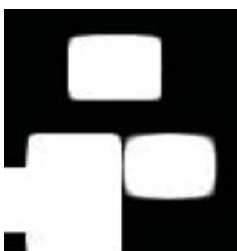

(c)

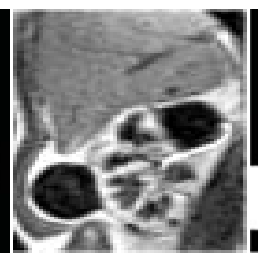

(d)

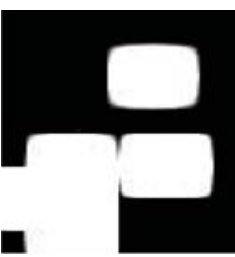

(e)

Fig. 1. (a) Kidney in one typical frame of pre-contrast stage; (b) saliency map generated by entropy-based method; (c) by neurobiological model; (d) kidney image in post-contrast stage; and (e) saliency map in post-contrast stage

immediate surroundings, is a relative quantity and is a good measure of establishing correspondence between regions in the pre- and post-contrast enhanced phases. From our experiments we observe that the saliency maps for the preand post- contrast stages are nearly identical, i.e., salient regions still remain salient after contrast enhancement. To demonstrate this, we display the saliency map of the kidney in the pre- and post-contrast stages in Fig. 1 (c) and (e), respectively. As shown, the two saliency maps are very close in spite of changes in image intensity due to the wash-in of the contrast agent. This suggests that the difference between saliency values of two corresponding regions is a good indicator of their similarity in the presence of contrast enhancement. Hence, it is desirable to facilitate the registration of dynamic renal MR images by exploiting the saliency information. For more details about the advantages of the neurobiology model over an entropy based approach refer [15].

\section{Method}

\subsection{Overview of Our Algorithm}

We model the displacement field of the elastic deformation as a Markov random field. MRF models use neighborhood relations between pixels of an image to model the interaction field. In registration of dynamic renal MR images where the kidney could have undergone elastic displacement, the correspondence between structure in two sets of images can be improved by including neighborhood information in the registration process. In our algorithm we use a combination of pixel intensity, saliency information and edge-orientation histograms to register dynamic contrast enhanced images. Integrating both saliency and edge information into the MRF framework ensures that the resulting matching is between pixels that are semantically as well as perceptually similar. Including saliency and intensity information from a neighborhood ensures smoothness of the deformation field. The displacements were denoted as discrete labels and the optimal labeling was determined by graph-cuts. 


\subsection{MRF Model for Nonrigid Registration}

The standard energy function in MRFs takes the following form

$$
E(x \mid \theta)=\sum_{s \in V} \theta_{s}\left(x_{s}\right)+\sum_{(s, t) \in N} \theta_{s t}\left(x_{s}, x_{t}\right)
$$

where $V$ is the set of pixels; $x_{s}$ denotes the label of pixel $s \in V ; N$ is a neighborhood system defined on $V$. The term $\theta_{s}$ is a unary data penalty function and $\theta_{s t}$ is a pairwise potential function that incorporates neighborhood information into the energy function.

We introduce an additional pairwise potential term $\theta_{\text {edge }}$, and define our energy function as

$$
E(x \mid \theta)=\sum_{s \in V} \theta_{s}\left(x_{s}\right)+\sum_{(s, t) \in N} \theta_{s t}\left(x_{s}, x_{t}\right)+\sum_{(s, t) \in N} \theta_{\text {edge }}\left(x_{s}, x_{t}\right) .
$$

In our experiments, the interaction potential is defined at the level of pixelblocks and the neighborhood information is collected over a window comprising of blocks of pixels. Therefore $s$ in equation (2) denotes blocks of pixels. We detail now each of the terms in equation (2).

\section{A. Unary Data Term: $\theta_{s}\left(x_{s}\right)$}

Let $x_{i}^{1}$ denote the pixel intensity in the floating image and $x_{i}^{2}$ the pixel intensity in the reference image. The data term is given by

$$
\theta_{s}\left(x_{s}\right)=\sum_{i=1}^{m} w_{i} \times\left(x_{i}^{1}-x_{i}^{2}\right)^{2}
$$

It is the sum-of-squared differences of pixel intensities for each pixel block in the floating image and the reference image weighted by a function of the saliency difference between the constituent pixels. The weight $w_{i}$ for the $i^{\text {th }}$ pixel in the pixel block is defined as

$$
w_{i}=\frac{\exp \left(\left|s v_{i}^{1}-s v_{i}^{2}\right|\right)}{1+\exp \left(\left|s v_{i}^{1}-s v_{i}^{2}\right|\right)}
$$

where $s v_{i}^{1}$ and $s v_{i}^{2}$ are the normalized saliency value of the corresponding pixel in the floating and reference image respectively. In equation (3), $m$ is the total number of pixels in each block, i.e., $m=n \times n$, where $n$ is the size of the square block.

\section{B. Pairwise Interaction Term: $\theta_{s t}\left(x_{s}, x_{t}\right)$}

Recall that $\theta_{s t}\left(x_{s}, x_{t}\right)$ is the interaction term between the block and its neighbors. Let $s+\left(x_{s}, x_{t}\right)$ be the block $s$ shifted by $\left(x_{s}, x_{t}\right)$. Therefore,

$$
\theta_{s t}\left(x_{s}, x_{t}\right)=\sum_{j} d\left(x_{s}, x_{s+\left(x_{s}, x_{t}\right)}\right),
$$


where $d\left(x_{s}, x_{s+\left(x_{s}, x_{t}\right)}\right)=\sum_{i=1}^{m} w_{i} \times\left|x_{i}^{1}-x_{i}^{2}\right|$ is the dissimilarity measure between blocks $s$ and $s+\left(x_{s}, x_{t}\right)$ and $j$ denotes all neighboring blocks. In our formulation, we constrain the range of allowed displacements by imposing a large penalty for blocks with a displacement greater than 6 pixel units from the central block.

Here we explain the rationale behind the weight function $w_{i}$ introduced in equation (3). The requirement for a weighting function was that it should assign low values to small difference in saliency and at the same time ensure that saliency information does not bias the energy term. To avoid dealing with very large numbers, a function is chosen so that its value lies between 0 and 1 . Our choice of the weighting function was determined empirically and is not unique. In the scenario that the blocks of pixels being compared belong to the same region, the difference in their normalized saliency values is very small and the corresponding $w_{i}$ is small. Also, the difference in intensity values is minimum for identical regions even in the face of intensity change due to wash-in of contrast agent. Thus the resultant product of the two terms is minimal.

When the two blocks in question are from different regions then the saliency values are different and a large difference results in a high value of $w_{i}$. This combined with the large intensity difference makes the terms $\theta_{s}\left(x_{s}\right)$ and $\theta_{s t}\left(x_{s}, x_{t}\right)$ in equation (2) have a higher value. There is the possibility that we might have pixel blocks from regions having significantly different intensities but similar saliency values. In that case, the product of $w_{i}$ and $\left|x_{i}^{1}-x_{i}^{2}\right|$ takes a high value and the terms $\theta_{s}\left(x_{s}\right)$ and $\theta_{s t}\left(x_{s}, x_{t}\right)$ are not minimal. In the normalized saliency map from the neurobiology based model, the salient regions are distinctly different from their neighbors. Thus, in a local neighborhood it is highly improbable to find pixels with identical or nearly identical values. In our experiments, the images have been registered for rigid transformations prior to nonrigid image matching. As a result, considering the amount of elastic motion a kidney could undergo, we constrain the range of allowed displacements for a pixel to \pm 6 pixel units in each direction. In such a setup, the scenario that pixel blocks being compared are from vastly different locations and have nearly identical saliency values does not arise.

\section{Edge Potential Term: $\theta_{\text {edge }}\left(x_{s}, x_{t}\right)$}

Elastic deformations in a kidney are like local rotations. As a potential term, $\theta_{\text {edge }}\left(x_{s}, x_{t}\right)$ is a rotation invariant metric that is used to match the edges of the object of interest, i.e., the kidney. We use edge-orientation histograms to define rotation invariant features [16] which are important for matching landmarks. The degree of dissimilarity between two sets of edges is determined by taking the histogram distance of the distribution of their orientations, as in equation (6). We observe that in the post-enhancement stage, the direction of edges at certain points is inverted due to increased intensity. In such a scenario, we ensured that gradient directions differing by $\pi$ radians are grouped in identical bins.

$$
\theta_{\text {edge }}\left(x_{s}, x_{t}\right)=\sum_{k=1}^{K}\left(h_{k}^{1}-h_{k}^{2}\right)^{2},
$$


where $h_{k}$ is the value in the $k^{t h}$ bin of edge orientation histogram $h$, and $K$ is the number of histogram bins. $K$ was set to 5 in our experiments.

\subsection{Optimization}

The range of displacements was denoted as a series of discrete labels and the optimal labeling determined the displacement of each pixel block. Optimization of equation (2) was carried out using graph-cuts, 9, via a sequence of alphaexpansion ( $\alpha$-expansion) moves. Details of the method can be found in 9 .

\section{Experimental Results}

\subsection{Implementation}

We tested our algorithm on 5 different datasets. The MR images of the kidney were of dimensions $256 \times 256$. There were 41 volumes acquired for each patient with each volume having 40 slices. The intensity values of the images and also that of the saliency map were normalized to lie in the range [0,1]. All images were pre-registered for rigid transformations before implementing our algorithm. Non-overlapping pixel blocks of size $3 \times 3$ were used.

\subsection{Results on Real Datasets}

In registration of contrast-enhanced MR images, the difference image between the pre- and post-contrast stages after registration should show only the renal tissues (i.e, renal cortex) whose intensity is enhanced due to the contrast agent. We first evaluate the registration results by visual examination. Fig. 2 (a) shows the unregistered difference image between a reference pre-contrast image and the floating post-contrast image. We can see the significant error due to elastic deformation. Fig. 2 (b) shows the difference image after our registration algorithm has been applied to the floating image without using saliency (by setting $w_{i}=1$ in equations (3) and (5)) or edge information. Fig. 2 (c) shows the difference image when using saliency information alone. The registration results are not optimum with some regions improperly registered. Fig. 2 (d) shows the difference image after edge information along with saliency information has been used in our registration algorithm. This image clearly shows that in spite of large intensity differences due to the wash-in of the contrast agent, our MRF based method accomplishes registration very effectively.

\subsection{Results of Simulated Deformation}

In each dataset, pre-contrast images were elastically deformed using the freeform deformation (FFD) method in 6]. These deformed images were then registered to the original undeformed image using our MRF based algorithm. The difference images for a typical pre-contrast image are shown in Fig. 3 (a)-(b). Fig. 3 (a) shows the difference image before registration and Fig. 3 (b) shows 


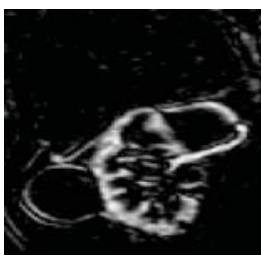

(a)

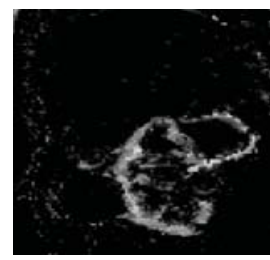

(b)

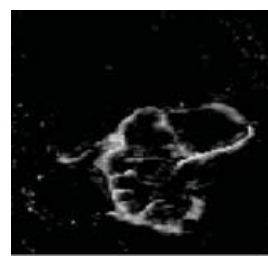

(c)

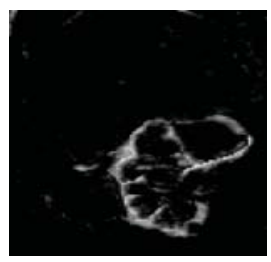

(d)

Fig. 2. Difference images: (a) before registration; after registration (b) using only intensity information; (c) saliency combined with intensity information; (d) using saliency and edge information together with intensity information

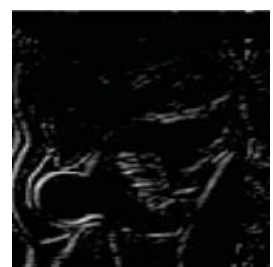

(a)

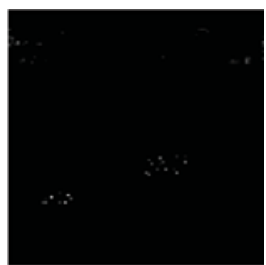

(b)

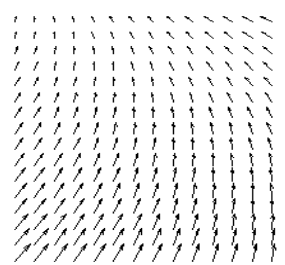

(c)

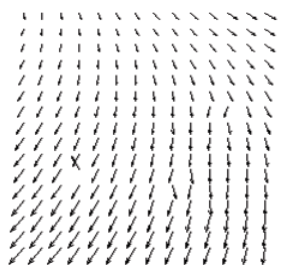

(d)

Fig. 3. Results of simulated elastic deformation (for pre-contrast stage): (a) difference image before registration; (b) difference image after registration; (c) simulated displacement field about the kidney region; and (d) recovered displacement field about the kidney region

the difference image after registration. From Fig. 3 (b) we conclude that our registration algorithm does well to recover the flow field. In Fig. 3 (c) we show the displacement field generated by applying the FFD algorithm and Fig. 3 (d) shows the displacement field recovered by our method. From the two images it is apparent that our algorithm could recover the simulated flow field with a considerable degree of accuracy. Similar results were obtained in our attempt to recover the flow field of a post-contrast image. The average error (Euclidean distance) between the actual deformation field and recovered flow field was found to be $\{0.0053,0.0125,0.0046,0.0061,0.0097\}$ units for the datasets.

\section{Conclusion}

In this paper we have proposed a saliency based MRF model for registration of dynamic contrast enhanced images in the presence of intensity changes due to flow of contrast agent. To obtain the saliency information we used a neurobiologybased saliency model that works better than an entropy-based saliency model. Difference of saliency values from a normalized saliency map combined with intensity information was used to quantify the degree of similarity between two regions at different time intervals. Information from these two maps was fused 
using an exponential function of the saliency difference such that it assigns low values to small differences in saliency and at the same time ensures that saliency information does not bias the energy term. In the formulation of the cost function of the MRF model we used the edge-orientation histogram to incorporate rotationally-invariant edge information. Tests on 5 real patient datasets with different degrees of elastic deformation of the kidneys, demonstrate the effectiveness and accuracy of our method. We simulated elastic deformations using FFD and the recovered displacement field obtained using our algorithm was very close to the inverse of the simulated field.

\section{Acknowledgements}

The authors would like to thank Dr. Vivian Lee, Professor of Radiology, Physiology and Neuroscience, Vice-Dean for Science, Senior Vice-President and Chief Scientific Officer, New York University Medical Center, for providing the datasets. This work was supported by NUS grant R-263-000-470-112.

\section{References}

1. Bajcsy, R., Kovacic, S.: Multiresolution elastic matching. Computer Vision, Graphics, and Image Processing 46(1), 1-21 (1989)

2. Christensen, G., Miller, M.I., Vannier, M.: 3D brain mapping using a deformable anatomy. Phy. Med. Biol. 39, 609-618 (1994)

3. Bro-Nielsen, M., Gramkow, C.: Fast fluid registration of medical images. In: Höhne, K.H., Kikinis, R. (eds.) VBC 1996. LNCS, vol. 1131, pp. 267-276. Springer, Heidelberg (1996)

4. Thirion, J.P.: Image matching as a diffusion process: an analogy with maxwell's demons. Med. Image Anal. 2(3), 243-260 (1998)

5. Meyer, C., et al.: Demonstration of accuracy and clinical versatility of mutual information for automatic multimodality image fusion using affine and thin-plate spline warped geometric deformations. Medical Image Analysis 1(3), 195-206 (1997)

6. Rueckert, D., Sonoda, L.I., Hayes, C., Hill, D.L., Leach, M.O., Hawkes, D.J.: Nonrigid registration using free-form deformations: application to breast $\mathrm{mr}$ images. IEEE Trans. Med. Imaging 18(8), 712-721 (1999)

7. Rohde, G.K., Aldroubi, A., Dawant, B.M.: The adaptive bases algorithm for intensity based nonrigid image registration. IEEE Trans. Med. Imaging 22(11), 1470 $1479(2003)$

8. Roy, S., Govindu, V.: Mrf solutions for probabilistic optical flow formulations. In: ICPR 2000: Proceedings of the International Conference on Pattern Recognition, p. $7053(2000)$

9. Boykov, Y., Veksler, O., Zabih, R.: Fast approximate energy minimization via graph cuts. IEEE Trans. Pattern Anal. Mach. Intell. 23(11), 1222-1239 (2001)

10. Shekhovtsov, A., Kovtun, I., Hlavác, V.: Efficient mrf deformation model for nonrigid image matching. In: CVPR. IEEE Computer Society, Los Alamitos (2007)

11. Tang, T.W.H., Chung, A.C.S.: Non-rigid image registration using graph-cuts. In: Ayache, N., Ourselin, S., Maeder, A. (eds.) MICCAI 2007, Part I. LNCS, vol. 4791, pp. 916-924. Springer, Heidelberg (2007) 
12. Zheng, Y., Yu, J., Kambhamettu, C., Englander, S., Schnall, M.D., Shen, D.: Deenhancing the dynamic contrast-enhanced breast mri for robust registration. In: Ayache, N., Ourselin, S., Maeder, A. (eds.) MICCAI 2007, Part I. LNCS, vol. 4791, pp. 933-941. Springer, Heidelberg (2007)

13. Kadir, T., Brady, M.: Saliency, scale and image description. International journal of Computer Vision 45(2), 85-105 (2001)

14. Itti, L., Koch, C.: A saliency-based search mechanism for overt and covert shifts of visual attention. Vision Research 40, 1489-1506 (2000)

15. Mahapatra, D., Sun, Y.: Registration of dynamic renal $\mathrm{mr}$ images using neurobiological model of saliency. In: Intl. Symp. Biomed. Imaging, pp. 1119-1122 (2008)

16. Lowe, D.G.: Distinctive image features from scale-invariant keypoints. Int. J. Comput. Vision 60(2), 91-110 (2004) 